\title{
Human Herpesvirus 6 Infection and Risk of Chronic Fatigue Syndrome: A Systematic Review and Meta-Analysis
}

\author{
Sayed-Hamidreza Mozhgania,b Farid Rajabic Mohsen Qurbanic \\ Yousef Erfani $^{d}$ Somayeh Yaslianifard $^{\text {b,e }}$ Azam Moosavi $^{f}$ \\ Kiomars Pourrostamig Ali Baradaran Bagheri ${ }^{\text {h }}$ Alireza Soleimani ${ }^{i}$ \\ Farida Behzadian ${ }^{j}$ Mahshid Safavi ${ }^{k}$ Farhad Rezaei ${ }^{\mathrm{c}}$
}

${ }^{a}$ Non-Communicable Diseases Research Center, Alborz University of Medical Sciences, Karaj, Iran; bepartment of Microbiology, Faculty of Medicine, Alborz University of Medical Sciences, Karaj, Iran; 'Department of Virology, School of Public Health, Tehran University of Medical Sciences, Tehran, Iran; ${ }^{d}$ Department of Medical Laboratory Sciences, School of Allied Medical Sciences, Tehran University of Medical Sciences, Tehran, Iran; eDietary Supplements and Probiotic Research Center, Alborz University of Medical Sciences, Karaj, Iran; fDepartment of Biochemistry, Faculty of Medicine, Alborz University of Medical Sciences, Karaj, Iran; 9Department of Pediatrics, School of Medicine, Alborz University of Medical Sciences, Karaj, Iran; hepartment of Neurosurgery, Madani Hospital, Alborz University of Medical Sciences, Karaj, Iran; 'Department of Infectious Disease, Alborz University of Medical Sciences, Karaj, Iran; 'Department of Bioscience and Biotechnology, Malek Ashtar University of Technology, Tehran, Iran; 'Student Research Center, Alborz University of Medical Sciences, Karaj, Iran

\section{Keywords}

Chronic fatigue syndrome · Human herpesvirus 6 .

Systematic review $\cdot$ Meta-analysis

\begin{abstract}
Introduction: Chronic fatigue syndrome (CFS) is a neurological disease that is accompanied by excessive fatigue or tiredness. There are several reports confirming the association between human herpesvirus 6 (HHV-6) infection and CFS illness. This systematic review and meta-analysis was performed to integrate the information of published studies with regard to this association until May 2021. Methods: The literature search was based on keywords including "chronic
\end{abstract}

karger@karger.com www.karger.com/int

Karger $\stackrel{\text { ' }}{5}$

GOPEN ACCESS
(C) 2021 The Author(s)

Published by S. Karger AG, Basel

This is an Open Access article licensed under the Creative Commons Attribution-NonCommercial-4.0 International License (CC BY-NC) (http://www.karger.com/Services/OpenAccessLicense), applicable to the online version of the article only. Usage and distribution for commercial purposes requires written permission. fatigue syndrome and HHV 6," "chronic fatigue syndrome and HHV-6," "chronic fatigue syndrome and HHV6," "chronic fatigue syndrome and Herpes virus 6," and "chronic fatigue syndrome and Herpesvirus6" in MEDLINE (PubMed), Web of Science, and EMBASE. Results: The literature search identified 17 studies to be included in the systematic review and 11 studies in meta-analysis. The symmetry funnel plot and Egger's test ( $p$ value $=0.2$ ) identified no publication bias among studies. Moreover, the low level of $P^{2}$ revealed homogeneity across studies. Discussion: In conclusion, the asso-

Sayed-Hamidreza Mozhgani and Farid Rajabi are co-first authors of the study.
Correspondence to:

Sayed-Hamidreza Mozhgani, hamidrezamozhgani@gmail.com Farhad Rezaei, rezaie@tums.ac.ir 
ciation between the HHV- 6 infection and CFS incidence was substantiated. However, the results of this study also suggest that further comprehensive studies are needed to solidify the association between HHV- 6 and CFS. Future studies should consider additional factors that may have affected the significance of such a correlation.

(c) 2021 The Author(s)

Published by S. Karger AG, Basel

\section{Introduction}

Neurological diseases are commonly referred to as central nervous system disorders that interfere with brain function, spinal cord, peripheral nerves, or muscle function. Chronic fatigue syndrome (CFS) stands among the most prevalent neurological diseases such as epilepsy, brain neoplasms, Alzheimer's, encephalitis, and meningitis. Chronic fatigue is a syndrome in which an affected person bears fatigue that does not recuperate with rest and sleep. It does not improve with rest and associates with physical symptoms. The Centers for Disease Control and Prevention (CDC) provided the following criteria to identify this syndrome: fatigue for $>6$ months, with at least 4 of the following symptoms: unrefreshing sleep, postexertional malaise, muscle pain, polyarthralgia, sore throat, tender lymph nodes, headache, and impaired memory or concentration [1].

CFS has a global distribution that affects individuals in all races and ages. There is no single diagnostic or biochemical test for this disease. The disease is usually diagnosed by excluding other diseases with fatigue as their main symptom according to the history, physical examination, and diagnostic tests of the patient [2]. The CDC considers infection, immune dysfunction, nutritional deficiency, and CNS dysfunction as possible causes of the disease. Several infectious agents could be associated with the syndrome [3]. A group of studies have focused on the role of immune system disorders as a primary cause of the CFS disease. Primary and secondary immunodeficiencies facilitate the emergence of opportunistic infections and result in insufficient control of persistent infections [4], which can trigger certain cases of CFS.

Human herpesvirus 6 (HHV-6) attacks the central nervous system and causes severe infections as well as replication in $\mathrm{T}$ cells which lead to the immune defense against a wide range of infections. Due to these unique characteristics, this virus may be one of the major contributors to CFS disease $[5,6]$.

HHV-6 belongs to the human herpesviridae family which is known to have latent infections in their hosts and to be reactivated intermittently, typically during periods of the relative immunosuppression [7]. HHV-6 has been associated with several adult diseases including various neurological disorders [8-11].

A systematic review provides an objective and reliable way to summarize research evidence. Through a systematic review, all studies on a specific research question are collected using explicit, transparent, and systematic methods to combine and interpret the results of studies with minimal bias and errors [12]. In some studies, there are reports of patients with CFS coinciding with large amounts of serum antibodies against HHV-6, hinting at a possible causal relationship between the infection and the syndrome.

In this study, we aimed to do a systematic review and meta-analysis on the relationships between the HHV-6 infection and development of CFS. The results of our study revealed the possible association between the HHV6 infection and incidence of the CFS disease in the literature.

\section{Materials and Methods}

\section{Search Strategy}

This study was performed in accordance with the PRISMA guidelines (see online suppl. Table 1 [PRISMA Checklist] at www. karger.com/doi/10.1159/000517930) [13]. All relevant studies were identified by the exploration of databases including MEDLINE (PubMed), Web of Science, and EMBASE by May 2021. Moreover, dissertations, scientific reports of congresses, and organization reports were explored as gray literature search. To find the related reports, the following keywords were used: "chronic fatigue syndrome and HHV 6, chronic fatigue syndrome and HHV-6, chronic fatigue syndrome and HHV6, chronic fatigue syndrome and Herpes virus 6, and chronic fatigue syndrome and Herpesvirus6." The systematic review was conducted in compliance with PRISMA statements [14]. Two independent reviewers screened and evaluated the studies. The agreement between authors to evaluate and select the articles was surveyed by calculating the kappa coefficient.

\section{Inclusion and Exclusion Criteria}

The inclusion criteria were studies that assessed the relationship between the HHV-6 infection with CFS incidence and the effect of antiviral treatment on the CFS treatment. Only the Englishlanguage articles were examined. However, the non-English articles with English-language abstracts were also included. The exclusion criteria were reviews, case-report studies, and in vitro studies using cell lines without clinical trial data.

Data Extraction and Quality Assessment

Two independent reviewers extracted data from the included studies through developing a data extraction sheet according to the rationale suggested by the Cochrane Consumers and Com-
Mozhgani et al. 
Fig. 1. Schematic flowchart of the conduct-

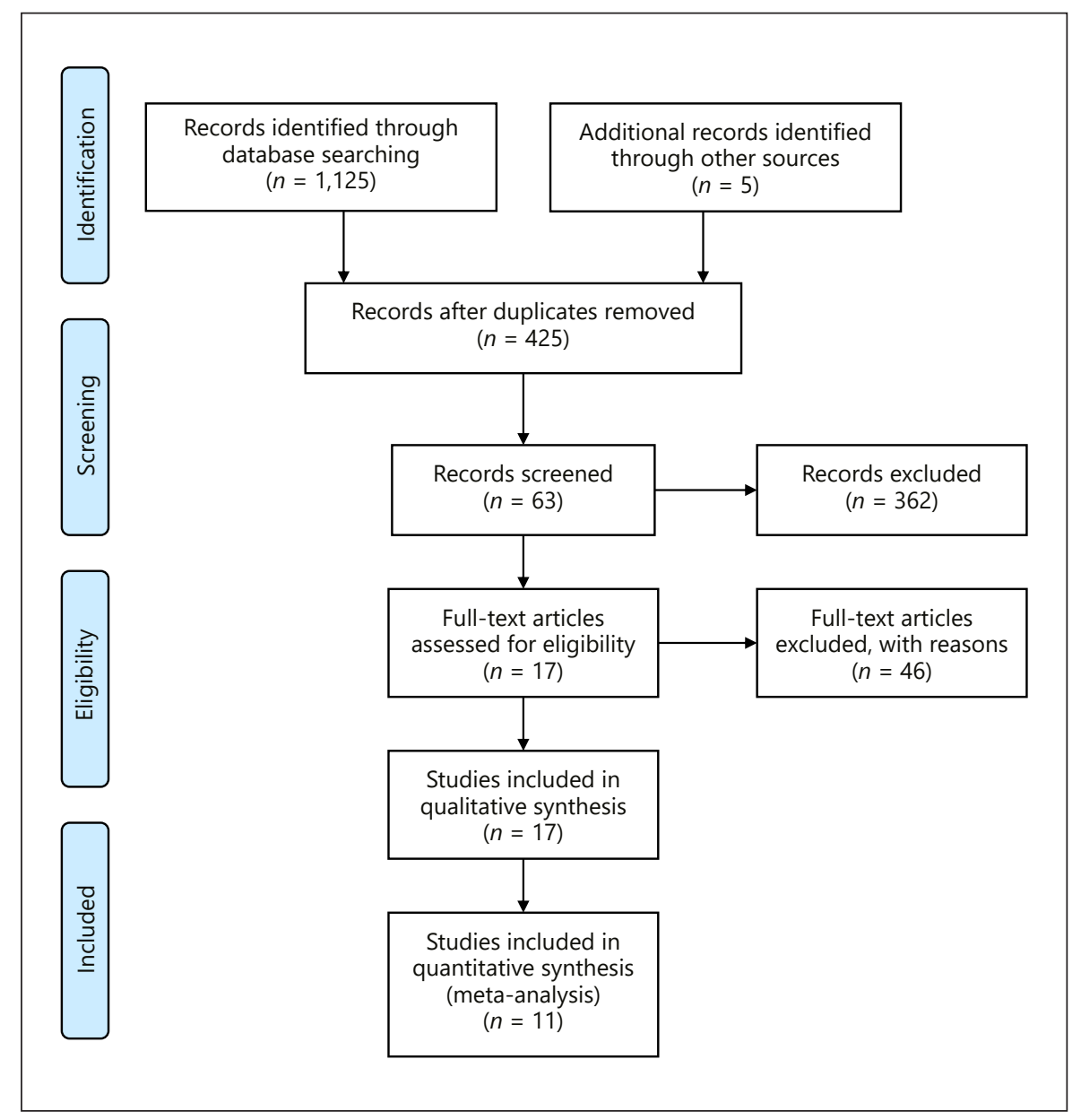
the PRISMA statements.

munication Group Data Extraction Template (available at http:// cccrg.cochrane.org/author-resources). The duplicate studies identified from various databases were considered only once. The details of each study including authors, year of publication, detection method, sample size, study, number of exposed/diseased cases (HHV-6+/CFS+), exposed/healthy cases (HHV-6+/CFS-), not exposed/diseased cases (HHV-6-/CFS+), not exposed/ healthy cases (HHV-6-/CFS-), and odds ratio were collected and considered at 3 levels: title, abstract, and full text. No disagreement was found between the study evaluations by the authors. The Newcastle-Ottawa Scale was employed to assess the quality and risk of bias. Three domains were considered to evaluate the risk of bias: (1) selection of case and control studies, (2) comparability of case and controls, and (3) ascertainment of exposure $[15,16]$. The score $\geq 6$ was considered as the high-quality score (low risk of bias), scores of $4-6$ as the moderate-quality score (moderate risk of bias), and scores $<4$ as the low-quality score (high risk of bias).

\section{Assessment of Publication Bias}

To assess the publication bias, a funnel plot was used in which an asymmetry plot displays possible publication bias. Egger's test was also performed to detect the asymmetry.

Herpesvirus 6 and Chronic Fatigue Syndrome
Assessment of Heterogeneity and Statistical Analysis

Meta-analysis and statistical analysis were performed using Cochrane review manager software (RevMan 5.3) through drawing a forest plot. Between-study heterogeneity was investigated using the $I^{2}$ test, since an $I^{2}$ of around $25 \%, 50 \%$, and $75 \%$ is taken into account as low, moderate, and high levels of heterogeneity, respectively. The pooled odds ratio and 95\% confidence interval were calculated using the Mantel-Haenszel method. The random-effects model was utilized for a high level of heterogeneity across the studies. Moreover, $\chi^{2}$ test was carried out to assess the statistical significance of the heterogeneity. The studies were divided into subgroups based on the detection method which they employed.

\section{Results}

\section{Literature Search and Study Characteristics}

The detailed steps of the search and studies selection are presented in Figure 1. The primary search identified 1,125 records in MEDLINE (426), Web of Science (322), 
Table 1. Summary details of the studies included in this meta-analysis [35-46]

\begin{tabular}{|c|c|c|c|c|c|c|c|c|}
\hline Author & $\begin{array}{l}\text { Detection } \\
\text { method }\end{array}$ & $\begin{array}{l}\text { Sample } \\
\text { size }\end{array}$ & $\mathrm{HHV}-6+/ \mathrm{CFS}+$ & $\mathrm{HHV}-6+/ \mathrm{CFS}-$ & HHV-6-/CFS+ & HHV-6-/CFS- & $\begin{array}{l}\text { Odds } \\
\text { ratio }\end{array}$ & $\begin{array}{l}\text { Quality } \\
\text { assessment }\end{array}$ \\
\hline Ablashi et al. [35] & Serology & 60 & 32 & 4 & 3 & 21 & 56 & $* * * * / * / * *$ \\
\hline Blomberg et al. [36] & Serology & 141 & 54 & 54 & 11 & 22 & 2 & $* * * * / * / * * *$ \\
\hline Burbelo et al. [37] & Serology & 131 & 61 & 53 & 11 & 6 & 0.69 & $* * * * / * * / * * *$ \\
\hline Cameron et al. [10] & PCR & 20 & 2 & 1 & 8 & 9 & 2.25 & $* * / * * / * * *$ \\
\hline Di Luca et al. [38] & PCR & 60 & 16 & 7 & 20 & 17 & 1.94 & $* * * * / * / * * *$ \\
\hline Enbom et al. [39] & PCR & 15 & 2 & 2 & 6 & 5 & 0.83 & $* * * / * / * *$ \\
\hline Knox and Carrigan [40] & PCR & 117 & 11 & 0 & 38 & 68 & 40.92 & $* * * * / * / * *$ \\
\hline Knox and Carrigan [40] & Serology & 25 & 4 & 56 & 51 & 136 & $5-69$ & $* * * * / * / * *$ \\
\hline Nicolson et al. [30] & PCR & 300 & 61 & 9 & 139 & 91 & 4.44 & $* * * * / * * / * * *$ \\
\hline Oakes et al. [41] & PCR & 48 & 16 & 4 & 23 & 5 & 0.87 & $* * * * / * * / * * *$ \\
\hline Patnaik et al. [31] & Serology & 319 & 93 & 7 & 61 & 145 & 31.58 & $* * * * / * / * * *$ \\
\hline Reeves et al. [42] & PCR & 76 & 7 & 14 & 19 & 36 & 0.95 & $* * * * / * / * * *$ \\
\hline Shikova et al. [43] & PCR & 108 & 48 & 41 & 10 & 9 & 1.05 & $* * * * / * / * * *$ \\
\hline Wallace et al. [44] & PCR & 145 & 26 & 19 & 48 & 52 & 1.48 & $* * * * / * / * * *$ \\
\hline Yalcin et al. [45] & Serology & 26 & 5 & 2 & 8 & 11 & 3.44 & $* * * * / * / * *$ \\
\hline Yalcin et al. [22] & PCR & 26 & 7 & 0 & 6 & 13 & 31.15 & $* * * * / * / * *$ \\
\hline Zorzenon et al. [46] & PCR & 103 & 30 & 6 & 22 & 45 & 10.23 & $* * * * / * / * * *$ \\
\hline
\end{tabular}

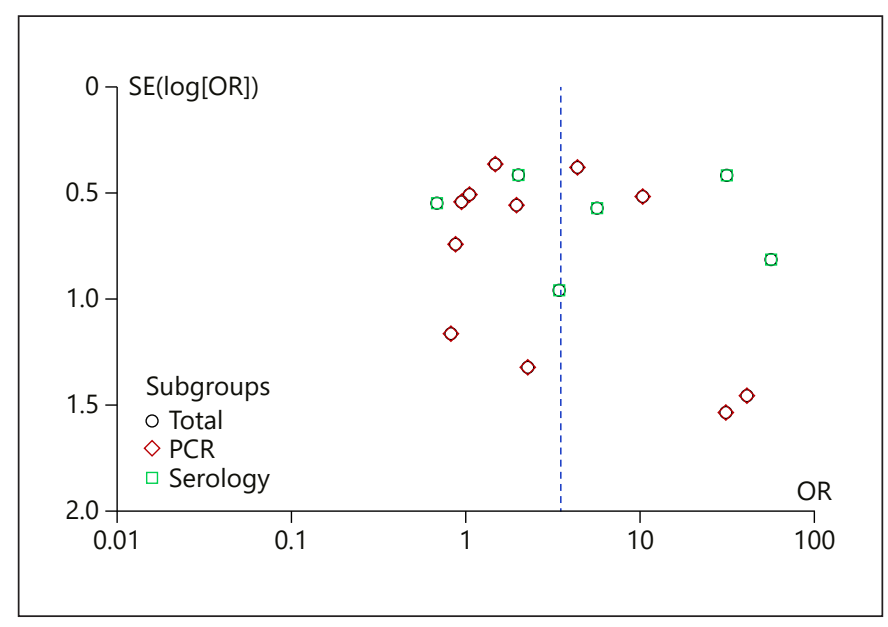

Fig. 2. Funnel plot of 17 studies for investigation of publication bias.

EMBASE (377), and gray literature search (5) based on the title screening. After removing duplicate reports, 425 studies were retained. Further screening at the title and abstract levels led to exclusion of 362 studies and inclusion of 63 studies. Finally, 17 studies (case-control studies) were evaluated at full-text level, and 46 studies were subsequently excluded. The characteristics of the selected studies are shown in Table 1. The kappa coefficient of 0.91 revealed the perfect agreement between 2 investigators.

\section{Quality Assessment}

The quality assessment of the studies according to the Newcastle-Ottawa Scale is summarized in Table 1. All of the 16 selected studies met the quality criteria since they earned at least 6 scores. Therefore, no study was excluded due to the low-quality score.

\section{Publication Bias and Heterogeneity}

In order to find the possible association between $\mathrm{HHV}$ 6 infection and CFS disease, the statistical analysis was performed, and the results were visualized on the forest plots. The funnel plot (Fig. 2) and Egger's test ( $p$ value $=$ 0.8 ) demonstrated no publication bias among studies. However, Figure 3 shows the significant heterogeneity across studies $\left(I^{2}=81 \%, \chi^{2}=84.66\right)$. The pooled odds ratio was obtained as 3.50 (95\% CI: 1.80-6.82). Moreover, we conducted 2 distinct meta-analyses of studies based on the detection method. The results showed again remarkable heterogeneity between studies which used PCR (pooled odds ratio $=2.43$ [95\% CI: 1.28-4.61], $I^{2}=64 \%$, $\chi^{2}=27.84$ ) and serology (pooled odds ratio $=5.89[95 \%$ CI: $1.48-23.45], I^{2}=89 \%, \chi^{2}=46.29$ ) methods. However, the pooled odds ratio suggests a significant association between the HHV-6 infection and risk of CFS. In the next analysis, 11 studies were selected to be involved in metaanalysis. The symmetric funnel plot (Fig. 4) and Egger's test $(p$ value $=0.2)$ demonstrated no publication bias among studies. The forest plot was first designed accord- 


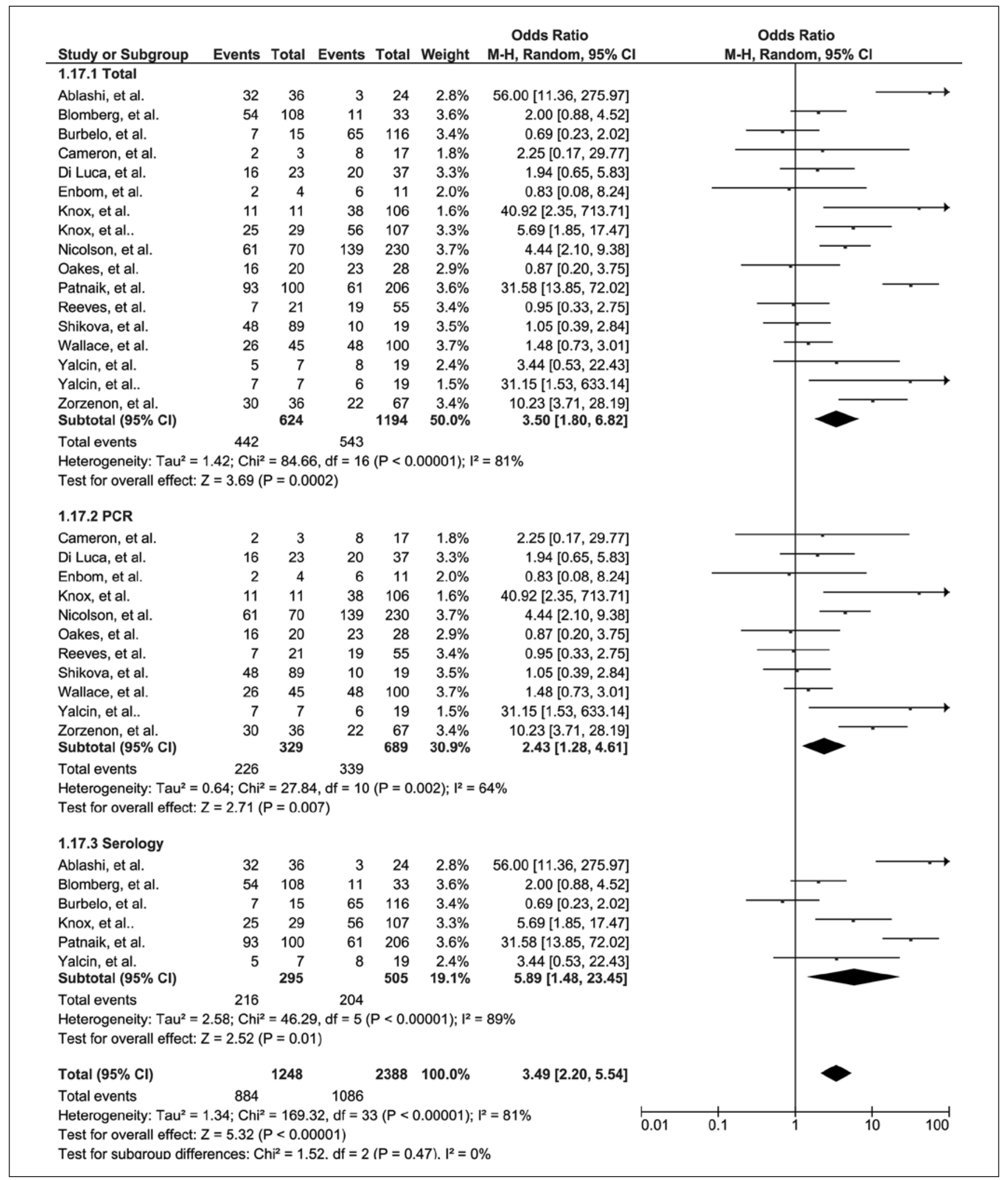

Fig. 3. Forest plot of the association between the HHV-6 infection and CFS illness based on 17 included studies. CFS, chronic fatigue syndrome; HHV-6, human herpesvirus 6.

Herpesvirus 6 and Chronic Fatigue Syndrome
Intervirology 2022;65:49-57 DOI: $10.1159 / 000517930$ 


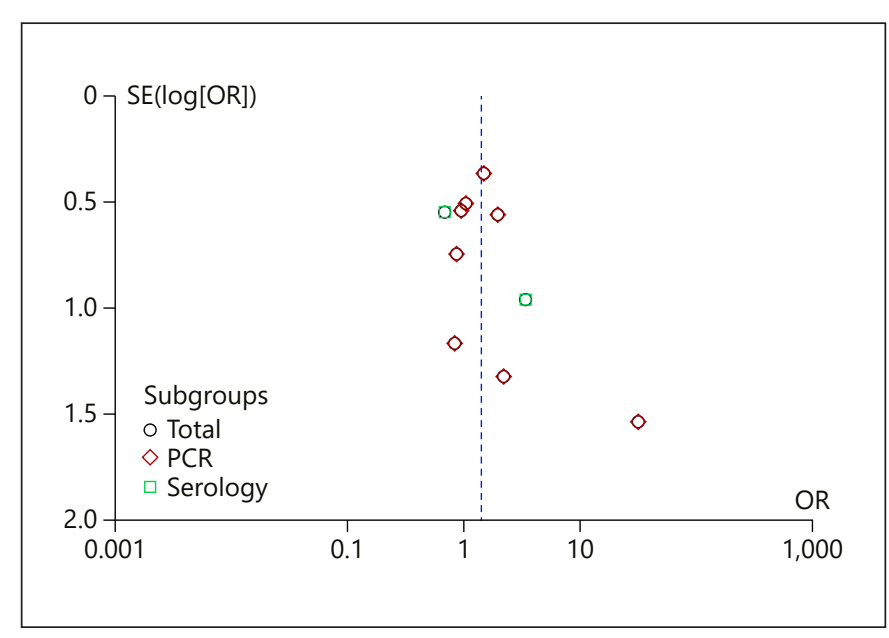

Fig. 4. Funnel plot of 11 studies for investigation of publication bias.

ing to all included studies. Figure 5 demonstrates the homogeneity across studies $\left(I^{2}=0 \%, \chi^{2}=9.38\right)$ with a pooled odds ratio of 1.4 (95\% CI: 0.99-1.99). Also, the subgroup analysis based on the detection method revealed no heterogeneity between studies (PCR: $I^{2}=0 \%, \chi^{2}=6.11$; serology: $\left.I^{2}=38 \%, \chi^{2}=3.25\right)$. The pooled odds ratio also suggests the possible association between HHV-6 infection and CFS incidence.

\section{Discussion}

HHV-6A is more frequently found in patients with neuroinflammatory diseases such as multiple sclerosis, whereas HHV-6B primary infection is the cause of exanthema subitum. Furthermore, HHV-6B reactivation is common in transplant recipients, leading to several clinical manifestations such as encephalitis, bone marrow suppression, and pneumonitis [17-19]. The present study provides the first systematic review and meta-analysis to evaluate the association between the HHV-6 infection and incidence of CFS. High between-study heterogeneity was first identified among 17 included studies, supposedly due to considerable differences in the design of studies including regions, sample size, and detection method. Moreover, there are some other factors that may be underestimated by the studies such as underlying diseases and infection by other viruses.

The systematic review and meta-analysis was carried out with 17 and 11 studies, respectively. The funnel plot analysis revealed no publication bias. The low amount of $I^{2}$ which determines the heterogeneity through calculat- ing total variation percentage clarified homogeneity across studies.

CFS usually begins with the infection by microorganisms or an immunological disorder [20,21]. Viral studies showed increased prevalence of 2 herpesviruses (HHV-6 and Epstein-Barr virus [EBV]) in the CFS subjects than the healthy ones $[20,22-25]$. Additionally, the possible association between infection by HHV- 6 and some diseases including Kikuchi-Fujimoto disease and systemic lupus erythematosus [26], autoimmune thyroid gland diseases [27], multiple sclerosis [28], and Rosai-Dorfman [29] has been reported. Despite the heterogeneity among studies, Nicolson et al. [30] and Patnaik et al. [31] studies employed a large sample size which can prove the possible relationship between HHV-6 infection and CFS. Nevertheless, further thorough studies are required.

Literature review showed several studies in which antiviral treatment was associated with the improvement of CFS symptoms. Larner et al. [32] investigated their proposal with regard to that HHV-6, EBV, and cytomegalovirus are the causative agents of CFS incidence. To evaluate this purpose, the treatment of patients who had been ill for a mean of 4.8 years with valacyclovir and/or valganciclovir for about 2.4 years was followed. The results disclosed that about $74.5 \%$ of the treated patients returned to a near-normal to normal life (primary endpoint). Therefore, they concluded that the CFS disease is due to the herpesvirus infection.

In another study, they suggested that the immediateearly gene expression in the myalgic encephalomyelitis ME/CFS patients causes host cell dysregulation and host cell apoptosis without lytic herpesvirus replication [33]. Treatment with certain antiviral nucleosides inhibits herpesvirus DNA polymerases while thymidine kinases act by inhibiting lytic virus replication. This results in suppression of the nonpermissive virus replication and may induce remission in ME/CFS patients.

Watt et al. [34] studied the association between the antibody titers against HHV-6 and EBV with the clinical response to valganciclovir in the CFS patients. In order to evaluate the response to treatment, self-reported physical and cognitive functioning levels were followed in the clinic visits. Fifty-two percent of the patients were recognized as responders, while $59 \%$ of them responded physically and $81 \%$ of them responded cognitively. However, no remarkable difference was detected in the change of antibody titers after treatment between the responder and nonresponder groups.

In this systematic review, several limitations may have affected the results of the study. First, there were limited 


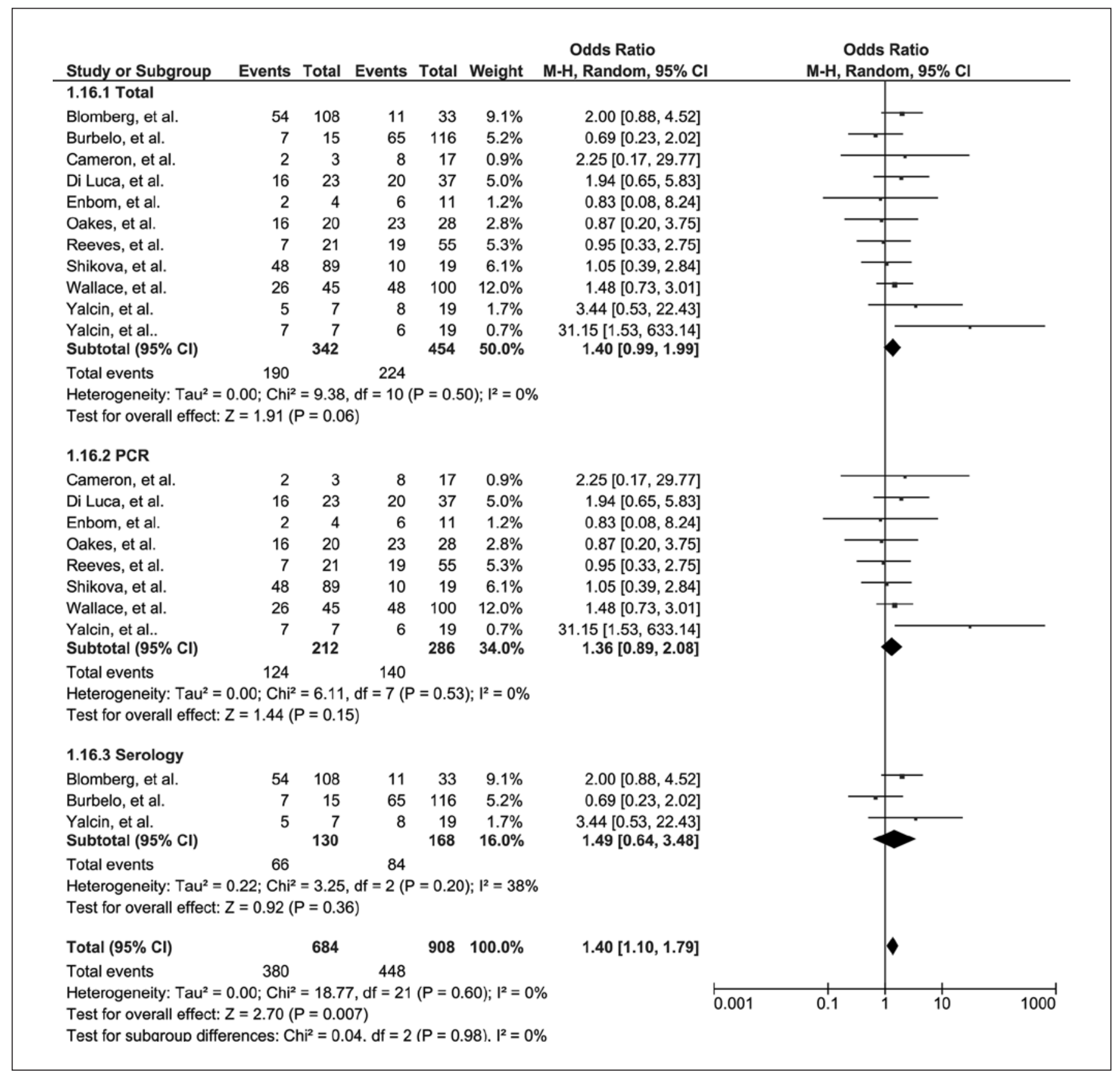

Fig. 5. Forest plot of the association between the HHV-6 infection and CFS illness based on 11 included studies. CFS, chronic fatigue syndrome; HHV-6, human herpesvirus 6.

reports that surveyed the association between HHV-6 and CFS. Second, the studies did not report the presence of other viruses. Third, distinct methods were utilized for virus detection in the included studies. The accumulated differences in study designs led to a considerable between-study heterogeneity. Further studies with large study population and sample size are recommended to provide robust evidence confirming the association between HHV-6 and CFS in humans while also considering other influencing factors such as the attendance of other viruses. 


\section{Acknowledgment}

The authors wish to thank the Alborz University of Medical Sciences, Iran, for supporting this work.

\section{Statement of Ethics}

This research was conducted ethically in accordance with the World Medical Association Declaration of Helsinki.

\section{Conflict of Interest Statement}

The authors declare no conflicts of interest relevant to this study.

\section{Funding Sources}

This work was funded by the Alborz University of Medical Sciences, Alborz, Iran.

\section{Author Contributions}

S.-H.M., F.R., M.Q., S.Y., A.B. and A.S. designed the project. S.-H.M., A.M. and Y.E. performed statistical analysis. S.-H.M., F.R., M.Q., K.P. and F.Re. interpreted and wrote the manuscript. F.R., M.Q. and M.S. did systematic search and literature review. All authors approved the final manuscript.

\section{References}

1 Yancey JR, Thomas SM. Chronic fatigue syndrome: diagnosis and treatment. Am Fam Physician. 2012 Oct 15;86(8):741-6.

2 Underhill RA. Myalgic encephalomyelitis, chronic fatigue syndrome: an infectious disease. Med Hypotheses. 2015 Dec;85(6):76573.

3 Komaroff A, Cho T. Role of infection and neurologic dysfunction in chronic fatigue syndrome. Semin Neurol. 2011;31(3):325-37.

4 Lum E, Medveczky MM, Medveczky PG. Is inherited human herpesvirus 6 the perpetrator behind some cases of chronic fatigue syndrome? Future Microbiol. 2014;9(4):433-6.

5 Yoshikawa T, Asano Y. Central nervous system complications in human herpesvirus-6 infection. Brain Dev. 2000;22(5):307-14.

6 Lusso P. HHV-6 and the immune system: mechanisms of immunomodulation and viral escape. J Clin Virol. 2006;37 Suppl 1:S4-10.

7 Hill JA, Venna N. Human herpesvirus 6 and the nervous system. Handb Clin Neurol. 2014; 123:327-55.

8 De Bolle L, Naesens L, De Clercq E. Update on human herpesvirus 6 biology, clinical features, and therapy. Clin Microbiol Rev. 2005 Jan;18(1):217-45.

9 Ablashi DV, Devin CL, Yoshikawa T, Lautenschlager I, Luppi M, Kühl U, et al. Review Part 3: human herpesvirus-6 in multiple non-neurological diseases. J Med Virol. 2010 Nov; 82(11):1903-10.

10 Cameron B, Flamand L, Juwana H, Middeldorp J, Naing Z, Rawlinson W, et al. Serological and virological investigation of the role of the herpesviruses EBV, CMV and HHV-6 in post-infective fatigue syndrome. J Med Virol. 2010 Oct;82(10):1684-8.

11 Yao K, Crawford JR, Komaroff AL, Ablashi DV, Jacobson S. Review part 2: human herpesvirus-6 in central nervous system diseases. J Med Virol. 2010 Oct;82(10):1669-78.
12 Tierney JF, Vale C, Riley R, Smith CT, Stewart $\mathrm{L}$, Clarke M, et al. Individual participant data (IPD) meta-analyses of randomised controlled trials: guidance on their use. PLoS Med. 2015 Jul;12(7):e1001855.

13 Moher D, Liberati A, Tetzlaff J, Altman DG; PRISMA Group. Preferred reporting items for systematic reviews and meta-analyses: the PRISMA statement. PLoS Med. 2009;6(7): e1000097.

14 Moher D, Liberati A, Tetzlaff J, Altman DG. Preferred reporting items for systematic reviews and meta-analyses: the PRISMA statement. Ann Intern Med. 2009;151(4):264-9, W64.

15 Peterson J, Welch V, Losos M, Tugwell P. The Newcastle-Ottawa scale (NOS) for assessing the quality of nonrandomised studies in meta-analyses. Ottawa: Ottawa Hospital Research Institute; 2011.

16 Wallis CJD, Saskin R, Choo R, Herschorn S, Kodama RT, Satkunasivam R, et al. Surgery versus radiotherapy for clinically-localized prostate cancer: a systematic review and meta-analysis. Eur Urol. 2016;70(1):21-30.

17 Yoshikawa T. Human herpesvirus 6 infection in hematopoietic stem cell transplant patients. Br J Haematol. 2004 Feb;124(4):42132.

18 De Bolle L, Van Loon J, De Clercq E, Naesens L. Quantitative analysis of human herpesvirus 6 cell tropism. J Med Virol. 2005 Jan;75(1): 76-85.

19 Alvarez-Lafuente R, Garcia-Montojo M, De las Heras V, Bartolome M, Arroyo R. Clinical parameters and HHV-6 active replication in relapsing-remitting multiple sclerosis patients. J Clin Virol. 2006 Dec;37(Suppl 1): S24-6.

20 Komaroff AL. Chronic fatigue syndromes: relationship to chronic viral infections. J Virol Methods. 1988 Sep;21(1-4):3-10.
21 Becker PD, McGregor N, Meirleirxy KD. Possible triggers and mode of onset of chronic fatigue syndrome. J Chronic Fatigue Syndr. 2002;10(2):3-18.

22 Yalcin S, Kuratsune H, Yamaguchi K, Kitani $T$, Yamanishi K. Prevalence of human herpesvirus 6 variants $A$ and $B$ in patients with chronic fatigue syndrome. Microbiol Immunol. 1994;38(7):587-90.

23 Jones JF, Ray CG, Minnich LL, Hicks MJ, Kibler R, Lucas DO. Evidence for active Epstein-Barr virus infection in patients with persistent, unexplained illnesses: elevated antiearly antigen antibodies. Ann Intern Med. 1985 Jan;102(1):1-7.

24 Josephs SF, Henry B, Balachandran N, Strayer D, Peterson D, Komaroff AL, et al. HHV-6 reactivation in chronic fatigue syndrome. Lancet. 1991 Jun 1;337(8753):1346-7.

25 Buchwald D, Cheney PR, Peterson DL, Henry B, Wormsley SB, Geiger A, et al. A chronic illness characterized by fatigue, neurologic and immunologic disorders, and active human herpesvirus type 6 infection. Ann Intern Med. 1992 Jan 15;116(2):103-13.

26 Hoffmann A, Kirn E, Kuerten A, Sander C, Krueger G, Ablashi D. Active human herpesvirus-6 (HHV-6) infection associated with Kikuchi-Fujimoto disease and systemic lupus erythematosus (SLE). In vivo. 1991;5(3):2659.

27 Sultanova A, Cistjakovs M, Gravelsina S, Chapenko S, Roga S, Cunskis E, et al. Association of active human herpesvirus-6 (HHV6) infection with autoimmune thyroid gland diseases. Clin Microbiol Infect. 2017;23(1):50. e1-e5.

28 Chapenko S, Millers A, Nora Z, Logina I, Kukaine R, Murovska M. Correlation between HHV-6 reactivation and multiple sclerosis disease activity. J Med Virol. 2003;69(1):1117. 
29 Levine PH, Jahan N, Murari P, Manak M, Jaffe ES. Detection of human herpesvirus 6 in tissues involved by sinus histiocytosis with massive lymphadenopathy (Rosai-Dorfman disease). J Infect Dis. 1992;166(2):291-5.

30 Nicolson GL, Gan R, Haier J. Multiple co-infections (Mycoplasma, Chlamydia, human herpes virus-6) in blood of chronic fatigue syndrome patients: association with signs and symptoms. APMIS. 2003 May;111(5):557-66.

31 Patnaik M, Komaroff AL, Conley E, Ojoamaize EA, Peter JB. Prevalence of Igm antibodies to human herpesvirus- 6 early antigen (P41/38) in patients with chronic fatigue syndrome. J Infect Dis. 1995 Nov;172(5):1364-7.

32 Lerner AM, Beqaj S, Fitzgerald J, Gill K, Gill C, Edington J. Subset-directed antiviral treatment of 142 herpesvirus patients with chronic fatigue syndrome. Virus Adapt Treat. 2010; 2(1):47-57.

33 Lerner M, Lerner S. A paradigm linking herpesvirus immediate-early gene expression apoptosis and myalgic encephalomyelitis chronic fatigue syndrome. Virus Adapt Treat. 2011;3(1):19-24.

34 Watt T, Oberfoell S, Balise R, Lunn MR, Kar AK, Merrihew L, et al. Response to valganciclovir in chronic fatigue syndrome patients with human herpesvirus 6 and Epstein-Barr virus IgG antibody titers. J Med Virol. 2012 Dec;84(12):1967-74.
35 Ablashi DV, Eastman HB, Owen CB, Roman MM, Friedman J, Zabriskie JB, et al. Frequent HHV-6 reactivation in multiple sclerosis (MS) and chronic fatigue syndrome (CFS) patients. J Clin Virol. 2000 May;16(3):179-91.

36 Blomberg J, Rizwan M, Böhlin-Wiener A, Elfaitouri A, Julin P, Zachrisson O, et al. Antibodies to human herpesviruses in myalgic encephalomyelitis/chronic fatigue syndrome patients. Front Immunol. 2019 Aug 14;10: 1946.

37 Burbelo PD, Bayat A, Wagner J, Nutman TB, Baraniuk JN, Iadarola MJ. No serological evidence for a role of HHV-6 infection in chronic fatigue syndrome. Am J Transl Res. 2012; $4(4): 443-51$.

38 Di Luca D, Zorzenon M, Mirandola P, Colle R, Botta GA, Cassai E. Human herpesvirus-6 and human herpesvirus-7 in chronic fatigue syndrome. J Clin Microbiol. 1995 Jun;33(6): $1660-1$.

39 Enbom M, Linde A, Evengård B. No evidence of active infection with human herpesvirus 6 (HHV-6) or HHV-8 in chronic fatigue syndrome. J Clin Microbiol. 2000 Jun;38(6):2457.

40 Knox KK, Carrigan DR. Active human herpesvirus six (HHV-6) infections in patients with chronic fatigue syndrome (CFS) and relapsing-remitting multiple sclerosis (RRMS). J Clin Virol. 2006 Dec;37:S116.
41 Oakes B, Hoagland-Henefield M, Komaroff AL, Erickson JL, Huber BT. Human endogenous retrovirus-K18 superantigen expression and human herpesvirus- 6 and human herpesvirus-7 viral loads in chronic fatigue patients. Clin Infect Dis. 2013 May;56(10):1394-400.

42 Reeves WC, Stamey FR, Black JB, Mawle AC, Stewart JA, Pellett PE. Human herpesviruses 6 and 7 in chronic fatigue syndrome: a casecontrol study. Clin Infect Dis. 2000 Jul;31(1): 48-52.

43 Shikova E, Reshkova V, Kumanova A, Raleva S, Alexandrova D, Capo N, et al. Cytomegalovirus, Epstein-Barr virus, and human herpesvirus- 6 infections in patients with myalgic encephalomyelitis/chronic fatigue syndrome. J Med Virol. 2020 Dec 1;92(12):3682-8.

44 Wallace HL 2nd, Natelson B, Gause W, Hay J. Human herpesviruses in chronic fatigue syndrome. Clin Diagn Lab Immunol. 1999 Mar; 6(2):216-23.

45 Yalcin S, Kuratsune H, Yamaguchi K, Kitani T, Yamanishi K. Prevalence of human herpesvirus 6 variants $A$ and $B$ in patients with chronic fatigue syndrome. Microbiol Immunol. 1994;38(7):587-90.

46 Zorzenon M, Rukh G, Botta GA, Colle R, Barsanti LA, Ceccherini-Nelli L. Active HHV-6 infection in chronic fatigue syndrome patients from Italy: new data. J Chronic Fatigue Syndr. 1996;2(1):3-12. 